\title{
Angular dependence of hysteresis shift in oblique deposited ferromagnetic/ antiferromagnetic coupled bilayers
}
A. B. Oliveira, R. L. Rodriguez-Suarez, S. Michea, H. Vega, A. Azevedo, S. M. Rezende, C. Aliaga, and J. Denardin

Citation: Journal of Applied Physics 116, 033910 (2014); doi: 10.1063/1.4890457

View online: http://dx.doi.org/10.1063/1.4890457

View Table of Contents: http://aip.scitation.org/toc/jap/116/3

Published by the American Institute of Physics

\section{Articles you may be interested in}

Tunable misalignment of ferromagnetic and antiferromagnetic easy axes in exchange biased bilayers Applied Physics Letters 100, 242406 (2012); 10.1063/1.4729040

Noncollinear ferromagnetic easy axes in $\mathrm{Py} / \mathrm{Ru} / \mathrm{FeCo} / \mathrm{lm}$ spin valves induced by oblique deposition Applied Physics Letters 104, 242404 (2014); 10.1063/1.4883886

The angular dependence of the exchange bias under the planar domain wall model AIP Advances 5, 057146 (2015); 10.1063/1.4921632

Non-linear interplay between exchange-bias-induced unidirectional anisotropy and oblique-deposition-induced uniaxial anisotropy

Journal of Applied Physics 114, 043911 (2013); 10.1063/1.4816622

Azimuthal angular dependent hysteresis loops of $\mathrm{Fe}_{50} \mathrm{Mn}_{50} / \mathrm{Ni}_{81} \mathrm{Fe}_{19}$ bilayers grown under a magnetic field Journal of Applied Physics 120, 175303 (2016); 10.1063/1.4966935

Defect-induced magnon scattering mechanisms in exchange-coupled bilayers Journal of Applied Physics 120, 223904 (2016); 10.1063/1.4971961

\section{Scilight} Sharp, quick summaries illuminating the latest physics research

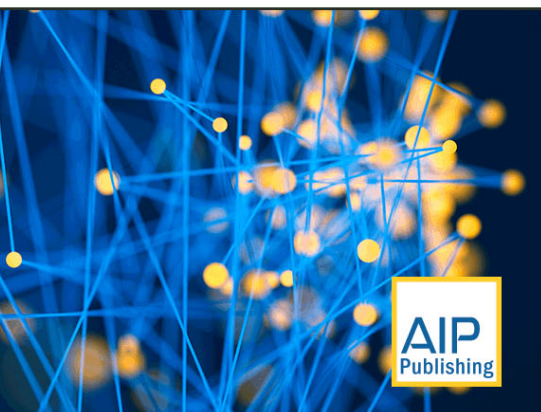




\title{
Angular dependence of hysteresis shift in oblique deposited ferromagnetic/ antiferromagnetic coupled bilayers
}

\author{
A. B. Oliveira, ${ }^{1,2}$ R. L. Rodriguez-Suarez, ${ }^{3}$ S. Michea, ${ }^{3}$ H. Vega,${ }^{3}$ A. Azevedo, ${ }^{4}$ \\ S. M. Rezende, ${ }^{4}$ C. Aliaga, ${ }^{5,6}$ and J. Denardin ${ }^{6,7}$ \\ ${ }^{1}$ Departamento de Física Teórica e Experimental, Univ. Fed. Rio Grande do Norte, BR-59072970 Natal, \\ $R N$, Brazil \\ ${ }^{2}$ Escola de Ciências \& Tecnologia Univ. Fed. Rio Grande do Norte, BR-59072970 Natal, RN, Brazil \\ ${ }^{3}$ Facultad de Física, Pontificia Universidad Católica de Chile, Av. Vicuña Mackenna 4860 Casilla 306, \\ Santiago, Chile \\ ${ }^{4}$ Departamento de Física, Universidade Federal de Pernambuco, 50670-901, Recife, Pernambuco, Brazil \\ ${ }^{5}$ Center for the Development of Nanoscience and Nanotechnology (CEDENNA), Avda. Ecuador 3493, \\ 9170124 Santiago, Chile \\ ${ }^{6}$ Facultad de Química y Biología, Universidad de Santiago de Chile, Casilla 40, Correo 33, Santiago, Chile \\ ${ }^{7}$ Departamento de Física, Universidad de Santiago de Chile (USACH), Avda. Ecuador 3493, \\ 917-0124 Santiago, Chile
}

(Received 2 June 2014; accepted 5 July 2014; published online 17 July 2014)

\begin{abstract}
The angular dependence of the hysteresis shift has been investigated in ferromagnetic/antiferromagnetic (NiFe/IrMn) bilayers grown by oblique deposition under the influence of a static magnetic field applied perpendicular to the uniaxial anisotropy direction induced during the growth process. It was found that at low oblique deposition angles, the unidirectional anisotropy field is much greater than the uniaxial anisotropy field and the corresponding anisotropies directions are noncollinear. In these conditions, the angular dependence of the hysteresis loop shift exhibits the well know cosine like shape but demanding a phase shift. Contrary to this, at high oblique deposition angle $\left(70^{\circ}\right)$, the uniaxial anisotropy plays the fundamental role and the anisotropies directions are collinear. In this case, the exchange bias displays a jump phenomenon. The numerical calculations are consistent with the experimental data obtained from magneto-optical Kerr effect and ferromagnetic resonance. (C) 2014 AIP Publishing LLC.
\end{abstract}

[http://dx.doi.org/10.1063/1.4890457]

\section{INTRODUCTION}

Fabrication of magnetic thin films by oblique deposition can enhance a wide variety of physical properties, such as magnetic anisotropy, magnetic coercivity fields, magnetic domain shape, resistivity, magnetostriction, and optical polarization. ${ }^{1-7}$ This enhancement is explained by modification of the film microstructure due to the growing conditions. During oblique deposition, columnar grains are growth tilted toward the source of particles. In addition, the grains are elongated perpendicular to the plane of incidence of particles and parallel to the plane of substrate. This phenomenon known as shadowing effect ${ }^{8}$ explains why magnetic thin films grown under this conditions exhibit a strong in-plane uniaxial anisotropy, with its easy axis perpendicular to the plane of incidence of sputtered atoms. On the other hand, the in-situ magnetic field, applied parallel to the substrate during sputtering deposition, can be used to break the magnetic symmetry, while the magnetic atoms are being deposited. Then, a magnetic anisotropy axis will be created parallel to the in-situ field. In this manner, deposition of magnetic thin films by oblique deposition under the influence of an inplane magnetic field can be used as a suitable procedure to investigate the role of induced anisotropies on the magnetic properties of the magnetic thin films and multilayers.

The samples studied in this work are essentially bilayer of antiferromagnetic (AF)/ferromagnetic (FM) materials that were grown at different oblique angles of deposition with an applied magnetic field perpendicular to the easy axis created by the shadowing effect (see Fig. 1(a)). Using this scheme, we can create a competition between the anisotropy induced by the applied magnetic field $\left(\boldsymbol{H}_{\text {grown }}\right)$ during deposition of the ferromagnetic layer and the anisotropy induced by the shadowing at oblique angles, giving rise to a difference in strength and direction between the exchange bias and uniaxial anisotropy field.

\section{EXPERIMENT}

The samples investigated in this work have the same multilayer structure $\mathrm{Cu}(3 \mathrm{~nm}) / \mathrm{Ir}_{20} \mathrm{Mn}_{80}(5 \mathrm{~nm}) / \mathrm{Py}(12 \mathrm{~nm}) /$ $\mathrm{Cu}(6 \mathrm{~nm}) /$ Substrate, and were deposited on commercial

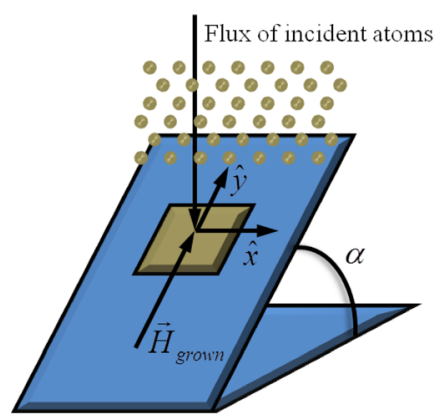

(a)

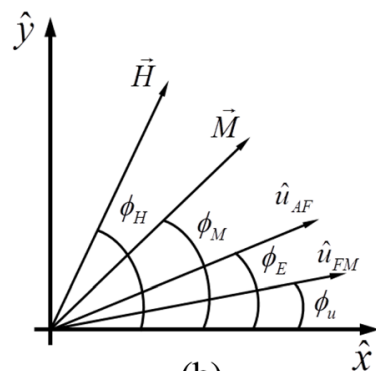

(b)
FIG. 1. (a) Illustration of sputtering oblique deposition set-up used to fabricate our samples. The magnetic field applied during growth is along the substrate plane. (b) Coordinate system used to write the free energy as a function of the in-plane angles. 
available $\mathrm{Si}(001)$ substrate by dc magnetron sputtering technique. A magnetic field $\left(\boldsymbol{H}_{\text {grown }}\right)$ of approximately 150 Oe was applied along the plane of the film during the deposition. Each sample was grown at different oblique angle $\left(\alpha=20^{\circ}\right.$, $30^{\circ}, 40^{\circ}, 50^{\circ}$, and $70^{\circ}$ ), as illustrated in Fig. 1(a). The base pressure was about $2.2 \times 10^{-7}$ Torr, and the working pressure was established introducing Ar gas inside the sputtering chamber until achieving 3.0 mTorr.

The magnetic properties of the samples was studied by the magneto-optical Kerr effect (MOKE) and ferromagnetic resonance (FMR). The MOKE measurements were carried out on a home-made set up. The intensity of a He-Ne $(632.8 \mathrm{~nm})$ laser beam $(10 \mathrm{~mW})$ was modulated by a chopper apparatus working at $1000 \mathrm{~Hz}$. The magnetic field was applied parallel to the film surface and the in-plane dependence of magnetization curves were measured using the MOKE longitudinal configuration. The FMR measurements were carried out on a Bruker EMX-1572 spectrometer $(9.8 \mathrm{GHz})$ at room temperature using a microwave cavity operating at $100 \mathrm{kHz}$ magnetic field modulation frequency. The sample was mounted on the tip of an external manual goniometer and placed at the center of the cavity so that the static magnetic field was perpendicular to the rf one and both parallel to the film surface. Such set up allows us to rotate the sample and obtain the in-plane angle dependence of resonance field determined by fitting the derivative of the absorption spectrum.

\section{MODEL}

Our theoretical approach is based on a phenomenological model that takes into account the relevant magnetic free energy terms. Considering the static and non perturbative behavior of the in-plane magnetization in longitudinal MOKE measurements, we can write the relevant free energy terms per unit area as

$$
\varepsilon=-\left[\boldsymbol{H} \cdot \boldsymbol{M}_{F M}+K_{u}\left(\frac{\boldsymbol{M}_{F M} \cdot \hat{\boldsymbol{u}}_{F M}}{M_{F M}}\right)^{2}\right] t_{F M}-J_{E} \frac{\boldsymbol{M}_{F M} \cdot \boldsymbol{M}_{A F}}{M_{F M} \cdot M_{A F}},
$$

where the first and second term are the Zeeman and uniaxial anisotropy energies of the FM layer, with uniaxial anisotropy constant $K_{u}$ and thickness $t_{F M}$. The last term in Eq. (1) represents the interfacial coupling energy of FM-AF bilayer, with exchange coupling constant $J_{E}>0$. Figure 1(b) shows the coordinate system used to represent the vectors of Eq. (1), where $\boldsymbol{H}, \boldsymbol{M}_{F M}$, and $\boldsymbol{M}_{A F}$ are the applied magnetic field, the magnetization of the FM layer, and the magnetization of the AF sublattice in atomic contact with FM, respectively. The unit vectors $\hat{\boldsymbol{u}}_{F M}$ and $\hat{\boldsymbol{u}}_{A F}$ represents the FM uniaxial anisotropy direction and $\mathrm{AF}$ pinning direction, respectively. We did not consider contributions from rotatable anisotropy because they can only be detected by means of perturbative techniques that are sensitive to the curvature of free energy. ${ }^{9,10}$ Here, we assumed a rigid planar domain wall in the AF so that $\boldsymbol{M}_{A F}$ is parallel to the pinning direction $\left(\boldsymbol{M}_{A F}=M_{A F} \hat{\boldsymbol{u}}_{A F}\right)$ and the AF domain wall energy does not contribute to our model.

In order to interpret the hysteresis loop data, obtained by means of the MOKE technique, Eq. (1) was solved by using the following energy minimization procedure. When magnetic field direction $\left(\phi_{H}\right)$ is set in a fixed direction and its strength is varied, the equilibrium position of the FM magnetization $\left(\phi_{M}^{0}\right)$ can be obtained from the first derivative condition

$$
\begin{aligned}
\left(\frac{\partial \varepsilon}{\partial \phi_{M}}\right)_{\phi_{M}=\phi_{M}^{0}}= & H \sin \left(\phi_{M}^{0}-\phi_{H}\right)+H_{E} \sin \left(\phi_{M}^{0}-\phi_{E}\right) \\
& +\frac{H_{u}}{2} \sin \left[2\left(\phi_{M}^{0}-\phi_{u}\right)\right]=0,
\end{aligned}
$$

where $H, H_{E}=\frac{J_{E}}{t_{F M} M_{F M}}$, and $H_{u}=\frac{2 K_{u}}{M_{F M}}$ are the applied magnetic field strength, exchange coupling field, and uniaxial anisotropy field, respectively. The angles in Eq. (2) are shown in Fig. 1(b). In addition to the condition give in Eq. (2), we also considered that $\left(\frac{\partial^{2} \varepsilon}{\partial \phi_{M}^{2}}\right)_{\phi_{M}=\phi_{M}^{0}}>0$, where $\phi_{M}^{0}$ represents the angle that minimizes Eq. (1).

In the process of films deposition, the FM layer was always deposited first than the AF one. As a result, the uniaxial anisotropy easy axis induced in FM layer is given by a combination of two mechanisms: (i) grain formation mechanism due to the oblique deposition and (ii) the application of the in-situ magnetic field during deposition. Both mechanisms have different microscopic contribution for the resultant FM layer anisotropy and they compete against each other as $\alpha$ varies from $20^{\circ}$ to $70^{\circ}$ (see Fig. 1(a)). As the AF atoms are being deposited on the FM layer, they are submitted to combined effects that control the resultant direction of the AF lattice magnetizations: (i) microscopic textures built on the FM surface due to the elongated grains; (ii) the superposition of the local field created by FM grains with the applied in-situ field; and (iii) the oblique deposition that itself might induces microstructrures characteristics of the IrMn. Therefore, we must to $\phi_{u} \neq \phi_{E}$ consider in Eq. (2).

Figure 2 shows hard axis hysteresis loops for samples grown at different oblique angles $\alpha=20^{\circ}, 40^{\circ}, 50^{\circ}$, and $70^{\circ}$. Observe that in the low field regime $M$ depends linearly with $H$, and crosses the origin with negligible coercivity. As one can see, the dependence of the hard axis direction as a function of the oblique deposition angle is not straightforward. However, it is clear that there will be always a direction, let it be $\phi_{H}=\phi_{H}^{*}$, in which hysteresis loops show a typical hard axis behavior. Using Eq. (2), for $\phi_{H}^{*}$ and considering the equilibrium position of the magnetization vector when $H=0\left(M \cos \left(\phi_{M}^{0}-\phi_{H}\right)=0\right.$ and $\left.H=0 \Rightarrow \phi_{M}^{0}=\phi_{H}^{*} \pm \frac{\pi}{2}\right)$, we obtain

$$
H_{E} \cos \left(\phi_{H}^{*}-\phi_{E}\right)-\frac{H_{u}}{2} \sin \left[2\left(\phi_{H}^{*}-\phi_{u}\right)\right]=0 .
$$

Equation (3) can be used to find out combinations of $H_{E}$, $H_{u}, \phi_{E}$, and $\phi_{u}$ to adjust the angular dependence of hysteresis loops shift measured by MOKE. However, we concluded that this procedure presents many possibilities resulting in good fits, but with no realistic values of the physical parameters. In order to accurate and realistic physical parameters from the data by using in Eq. (3), we incorporated results extracted from FMR measurements. Using a similar approach, but taking into account the dynamic behavior of the FM magnetization in FMR, it is also possible to extract $H_{u}, H_{E}, \phi_{u}$, and $\phi_{E}$ values as shown in Ref. 11. It is well 


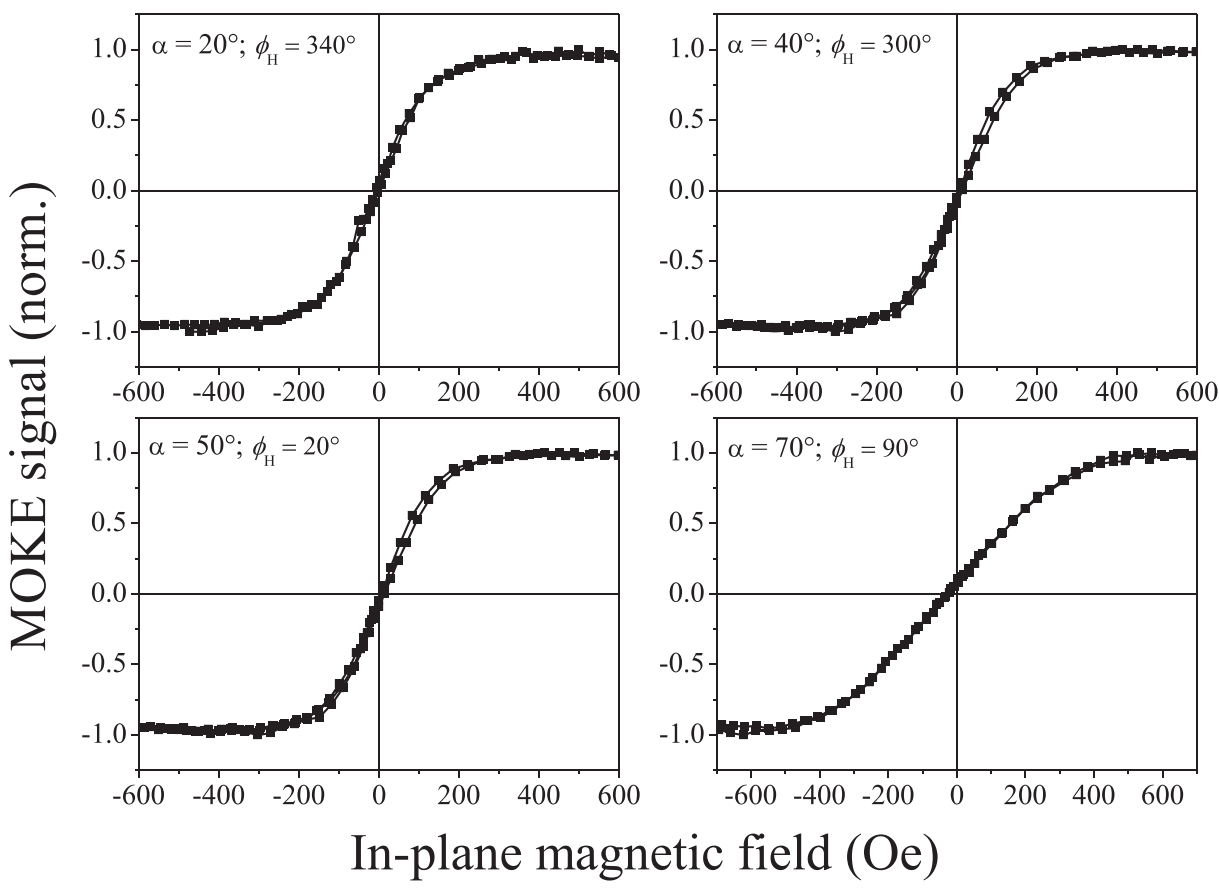

FIG. 2. Hysteresis loops with applied magnetic field parallel to the hard axis for FM/AF coupled bilayers. The inset angles are illustrated in Fig. 1.

known that FM/AF exchange coupling field values $\left(H_{E}\right)$ depend on the measurement technique used to investigate the phenomenon. ${ }^{9,12-15}$ In order to obtain the $H_{E}^{M O K E}$ value, we used $H_{u}, \phi_{u}$, and $\phi_{E}$ values close to those obtained by FMR in Eq. (3), then we compared the theoretical angular dependence of hysteresis loops shift to the experimental one.

\section{RESULTS AND DISCUSSION}

Figure 3 shows the angular dependence of hysteresis loops shift (as an example, see the hysteresis loop in the inset) for different oblique deposition angles $(\alpha)$. The zero value of the in- plane angle corresponds to the case in which the magnetic field is applied parallel to $\hat{x}$ defined in Fig. 1(a). In order to obtain the theoretical hysteresis loops shift, we numerically solved Eq. (2) taking into account the magnetic history of energy minimum and obtained a simulated hysteresis loop for each applied magnetic field direction $\left(\phi_{H}\right)$. The shift is defined as $\left(H_{c 1}+H_{c 2}\right) / 2$, where $H_{c 1}$ and $H_{c 2}$ are the switching fields at the descending and ascending branches of the hysteresis loop, respectively. As we can see, for $\alpha=20^{\circ}, 30^{\circ}, 40^{\circ}$, and $50^{\circ}$ independently of the misalignment between the uniaxial and unidirectional axes, the unidirectional anisotropy is dominant over the uniaxial anisotropy $\left(6.6<H_{E}^{M O K E} / H_{U}^{M O K E}<6.9\right)$. In this
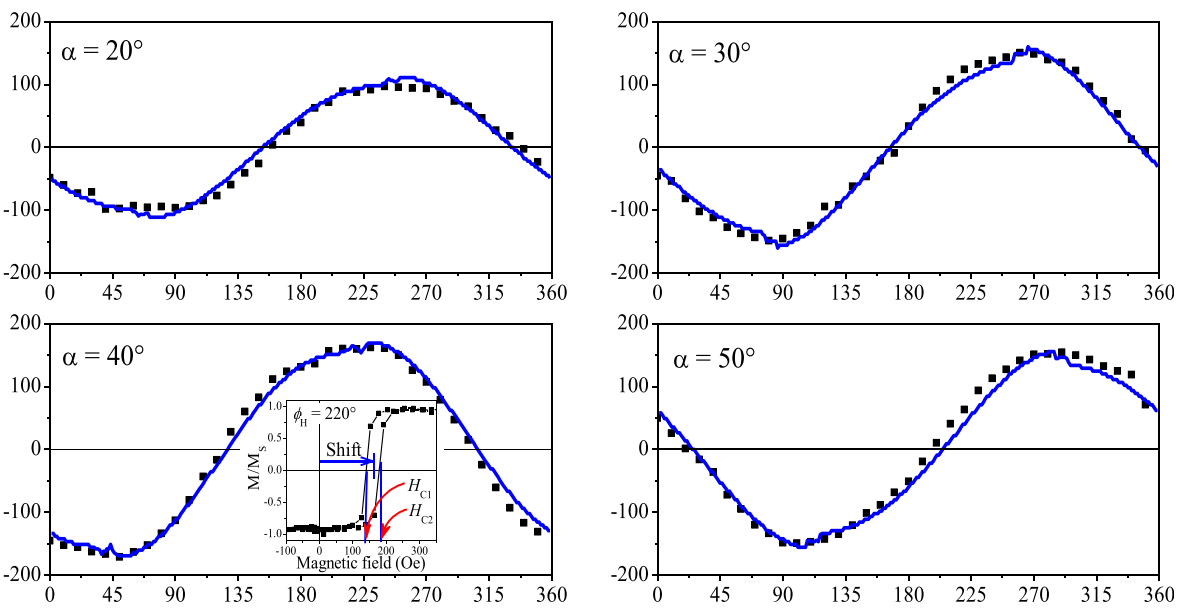

FIG. 3. Angular dependence of hysteresis loops shift. The square symbols are experimental results measured by MOKE and the solid lines are theoretical results obtained with our phenomenological model.

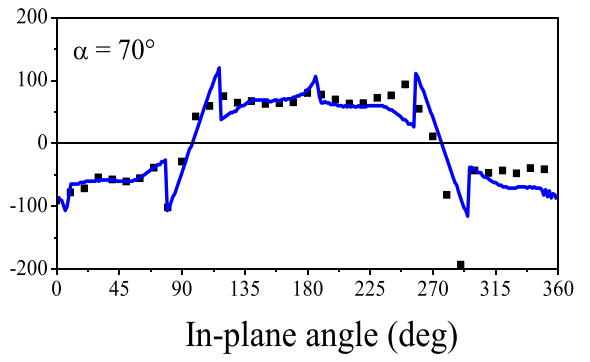




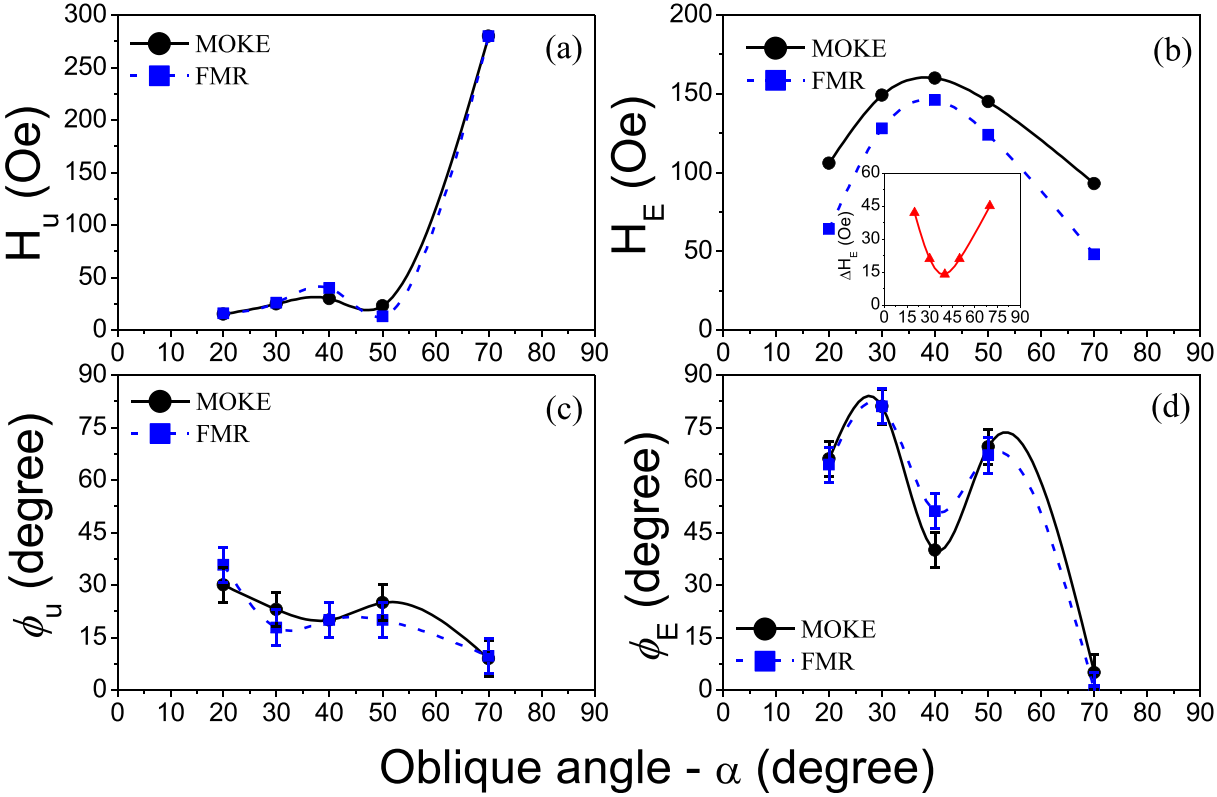

FIG. 4. Comparative results between FMR and MOKE data as a function of oblique deposition angle. The solid and dashed lines are guide to the eyes. (a) Uniaxial anisotropy field strength; (b) FM/AF exchange coupling field strength; (c) FM easy axis direction; and (d) AF pinning direction. case, the system will be in a monostable state in which, when the applied field is absent, only one energy minimum together with one energy maxima are presented in the dependence of the normalized energy $\frac{E}{2 K_{u} t_{F M}}$ on the FM magnetization orientation $\phi_{M} \cdot{ }^{13}$ In such state, $H_{c 1}$ and $H_{c 2}$ change continuously in the whole angular range $0^{\circ} \leq \phi_{H} \leq 360^{\circ}$ and consequently there are no jumps in the angular variation of the hysteresis loops shift exhibiting its well-known cosine like shape, but demanding a phase shift. For $\alpha=70^{\circ}$, where $H_{E}^{M O K E} / H_{U}^{M O K E} \cong 0.3$, the uniaxial anisotropy is dominant and the system will be in a bistable state, in which two stable magnetization-equilibrium orientations exist and both $H_{c 1}$ and $H_{c 2}$ are no more continuous in the angular range $0^{\circ} \leq \phi_{H} \leq 360^{\circ}$. This explains the jump in the angular dependence of the exchange bias showed in Fig. 3, for $\alpha=70^{\circ}$. $^{16-18}$ The magnetic parameter values used for fitting experimental results in Fig. 3 are shown in Fig. 4 as a function of oblique angle, as well as the values obtained from the FMR data.

Values of the uniaxial anisotropy field $\left(H_{u}\right)$ obtained from FMR and MOKE as a function of the oblique deposition angle $\alpha$ are shown in Figure 4(a). The dependence of $H_{u}$ with the oblique angle deposition shows a strong variation for $\alpha>50^{\circ}$ reaching a maximum value of 280 Oe at $\alpha=70^{\circ}$. This expected behavior is a result of the change in film microstructure as far as $\alpha$ increases. ${ }^{19,20}$ In addition, data from both MOKE and FMR show that $H_{u}$ presents a small hump for $\alpha$ around $40^{\circ}$.

Fig. 4(b) shows the dependence of the exchange field strength $\left(H_{E}\right)$ measured by MOKE and FMR on the oblique angle deposition. As expected $H_{E}$ values depend on measurement techniques and confirms that $H_{E}^{M O K E}>H_{E}^{F M R}$, as previously reported..$^{9,12-15}$ The fact that $H_{E}$ measured by MOKE is higher than the one measured by FMR can be explained in terms of the rotatable anisotropy. ${ }^{9,10}$ In FMR technique, applied magnetic field of the order of $\mathrm{kOe}$ is strong enough to saturate the magnetization and to rotate the antiferromagnetic spins of the low energy AF grains, creating an effective magnetic field named rotatable field. These rotated grains are called unstable grains. As a consequence, only non rotated grains (stable grains) will contribute for AF pinning direction and so for the $H_{E}$ strength. In the MOKE technique, $H_{E}$ measurement is based on field shift of hysteresis loops, where applied magnetic field is lower than the saturated one. In this case, the AF grains distribution is partially modified because the applied field strength is not capable to rotate all low energy AF grains pinning direction. These low energy and non rotated $\mathrm{AF}$ grains are equally responsible for $\mathrm{FM} / \mathrm{AF}$ interfacial exchange coupling as the AF stable grains. Therefore, contributions for AF pinning direction in MOKE experiment come from stable and some unstable AF grains (that were not rotated by the low applied field). As a consequence, $H_{E}$ strength has higher value in MOKE than in FMR. The inset of Fig. 4(b) shows difference between $H_{E}$ measured by MOKE and FMR, $\Delta H_{E}=H_{E}^{M O K E}-H_{E}^{F M R}$, as a function of the oblique angle deposition. It can assume percentage values that vary from $9 \%$ up to $48 \%$ in the $20^{\circ}<\alpha<70^{\circ}$ range. The difference in $H_{E}$ strength for the different angles showed in Fig. 4(b) reveals that the size distribution of AF grains is strongly affected by the oblique deposition technique. We might conclude that around $\alpha=40^{\circ}$, the film texture created by oblique deposition combined with the in-situ applied field favored the formation of AF stable grains resulting in a higher $H_{E}$ value and a lower $\Delta H_{E}$. As we note, for MOKE and FMR techniques, $H_{E}$ value does not exhibit the same overshoot as $H_{u}$ at high angle $\left(\alpha=70^{\circ}\right)$. This is an expected result since at high angles the same shadowing effect responsible for the increase of $H_{u}$ increases the roughness ${ }^{6}$ and as a direct consequence, $H_{E}$ decreases.

Regarding to the uniaxial anisotropy easy axis direction $\left(\phi_{u}\right)$, shown in Fig. 4(c), we observed a small fluctuation plus a monotonic decreasing as $\alpha$ increases. As expected, for high values of $\alpha$ more relevant and critical is the shadowing effect, causing the alignment of $H_{u}$ with $\hat{x}$, as shown in Fig. 1 .

Fig. 4(d) shows the dependence of the AF pinning direction $\left(\phi_{E}\right)$ as a function of the oblique angle deposition. It exhibits a similar behavior as $\phi_{u}$, for high $\alpha$ values the 
effective field due to the grain texture created in FM film is more relevant than the externally applied field, $\boldsymbol{H}_{\text {grown }}$.

\section{CONCLUSIONS}

In summary, we have studied the angular dependence of the hysteresis loop shift in oblique deposited FM/AF coupled bilayers in presence of an applied field $\boldsymbol{H}_{\text {grown }}$ perpendicular to the easy axis created by the shadowing effect. Under this scheme, we can induce a competition between the anisotropy created by $\boldsymbol{H}_{\text {grown }}$ and the shape anisotropy created by the shadowing effect. This competition gives rise to a difference in the strength and direction between the uniaxial and unidirectional anisotropies. We found that when the deposition angle is less than $50^{\circ}$, the unidirectional anisotropy is dominant over the uniaxial one, the system is in a monostable state, and the angular dependence of the hysteresis loop shift exhibits the well know cosine like shape but demanding a phase shift. On the other hand, when the deposition angle is high, the uniaxial anisotropy is dominant and the bilayer is in the bistable state. In this condition, the exchange bias field displays the jump phenomenon. We also propose that at intermediate angles, $\alpha \approx 40^{\circ}$, the film texture created by oblique deposition can favored the formation of $\mathrm{AF}$ stable grains resulting in a higher $H_{E}$. Furthermore, we found that for high $\alpha$ values the grain texture created in FM film is more relevant than $\boldsymbol{H}_{\text {grown }}$ and the AF pinning direction $\left(\phi_{E}\right)$ and uniaxial anisotropy easy axis direction $\left(\phi_{u}\right)$ are aligned.

\section{ACKNOWLEDGMENTS}

Research conducted in Recife was supported by the agencies CNPq (480724/2012-1), CAPES, FINEP, and
FACEPE (APQ-1292-1.05/10) and in Chile by the FONDECYT Grant Nos. 1140195, 1110736, 3130397, and 1130705 and Grant ICM P10-061-F by Fondo de Innovación para la Competitividad-MINECON.

${ }^{1}$ R. H. Welch and D. E. Speliotis, J. Appl. Phys. 41, 1254 (1970).

${ }^{2}$ M. M. Hawkeye and M. J. Brett, J. Vac. Sci. Technol., A 25, 1317 (2007).

${ }^{3}$ A. Lisfi and J. C. Lodder, Phys. Rev. B 63, 174441 (2001).

${ }^{4}$ S. van Dijken, G. Di Santo, and B. Poelsema, Phys. Rev. B 63, 104431 (2001).

${ }^{5}$ M. Mansour, A. S. Keita, B. Gallas, J. Rivory, A. Besnard, and N. Martin, Opt. Mater. 32, 1146 (2010).

${ }^{6}$ T. Otiti, G. A. Niklasson, P. Svedhlindh, and C. G. Granqvist, Thin Films Solids 307, 245 (1997).

${ }^{7}$ J. B. S. Mendes, L. H. V. Leão, S. M. Rezende, and A. Azevedo, IEEE Trans. Magn. 46, 2293 (2010).

${ }^{8}$ D. O. Smith, M. S. Cohen, and G. P. Weiss, J. Appl. Phys. 31, 1755 (1960).

${ }^{9}$ R. D. McMichael, M. D. Stiles, P. J. Chen, and W. F. Egelhoff, Phys. Rev. B 58, 8605 (1998).

${ }^{10}$ M. D. Stiles and R. D. McMichael, Phys. Rev. B 59, 3722 (1999).

${ }^{11}$ R. L. Rodríguez-Suárez, L. H. Vilela-Leão, P. Landeros, T. Bueno, S. M. Rezende, A. B. Oliveira, and A. Azevedo, Phys. Rev. B 83, 224418 (2011).

${ }^{12}$ H. Xi, R. M. White, and S. M. Rezende, J. Appl. Phys. 87, 4960 (2000).

${ }^{13}$ J. R. Fermin, M. A. Lucena, A. Azevedo, F. M. de Aguiar, and S. M. Rezende, J. Appl. Phys. 87, 6421 (2000).

${ }^{14}$ R. L. Rodríguez-Suárez, L. H. Vilela Leão, F. M. Aguiar, S. M. Rezende, and A. Azevedo, J. Magn. Magn. Mater. 272-276, 1212 (2004).

${ }^{15}$ J. Geshev, L. G. Pereira, and J. E. Schmidt, Phys. Rev. B 64, 184411 (2001).

${ }^{16}$ Y. Bai, G. Yun, and N. Bai, J. Appl. Phys. 107, 033905 (2010).

${ }^{17}$ Y. Bai, G. Yun, and N. Bai, J. Appl. Phys. 106, 063919 (2009).

${ }^{18}$ H. Yang and Y. Bai, Physica B 433, 12 (2014).

${ }^{19}$ R. D. McMichael, C. G. Lee, J. E. Bonevich, P. J. Chen, W. Miller, and W. F. Egelhoff, Jr., J. Appl. Phys. 88, 5296 (2000).

${ }^{20}$ G. Wang, C. Dong, W. Wang, Z. Wang, G. Chai, C. Jiang, and D. Xue, J. Appl. Phys. 112, 093907 (2012). 